\title{
ABOUT SOME PECULIARITIES OF EXPRESSIVE SYNTAX IN ENGLISH POPULAR PRESS
}

\author{
A.V. Mikhalcheva \\ Tula State Lev Tolstoy Pedagogical University, \\ 125, Lenin prospect, Tula, 300026, Russian Federation
}

\begin{abstract}
The article is devoted to the problems of expressive syntax and its role in transmitting the author's communicative intentions. According to the dominating cognitive-discourse paradigm at present, scientists are interested in researching the manifestations of the author's perception of transmitted information and their impact on the form of its presentation. Due to the dominating role of mass media in the contemporary world, it seems of great importance to reveal the way they influence the collective mind of the readers. In this connection expressive syntax plays a very important role in the process of communication via media text. The main aim of the research is to analyze functional and linguistic peculiarities of expressive syntax in media texts as a means of the author-reader interaction. In this article the research of the expressive syntax means is conducted on the basis of two English magazines. The topics and cognitive peculiarities of the readers play a great role in choosing proper expressive means and are required to be considered as a system. The results of the analysis show that the main means of expressive syntax in popular press is a parenthetical phrase serving to give the author's comments, define terms and toponyms, or add extra information, satisfying the readers' curiosity. The result also shows that due to different cognitive attitudes, types of creation (individual or collective) and segments of its targeted audience, National Geographic articles, unlike The Economist ones, contain more variable means of expressive syntax.
\end{abstract}

Key Words: expressivity, expressive syntax, media discourse, media text, popular press, and parenthetical phrase

For citation: Mikhalcheva A.V. 2021. About Some Peculiarities of Expressive Syntax in English Popular Press . Philological Sciences at MGIMO.Vol. 7. No 1(25). P. 60-67. https://doi.org/10.24833/24102423-2021-1-25-60-67

\section{О ПРАГМАТИЧЕСКИХ ОСОБЕННОСТЯХ ЭКСПРЕССИВНОГО СИНТАКСИСА В ТЕКСТАХ АНГЛОЯЗЫЧНОЙ ПОПУЛЯРНОЙ ПРЕССЫ}

\author{
А.В. Михалчева \\ Тульский государственный педагогический университет им. Л.Н. Толстого, \\ 300026, г. Тула, пр. Ленина, 125
}


Аннотация. Статья посвящена проблемам экспрессивного синтаксиса и роли данной категории в передаче коммуникативных интениий автора. Согласно главенствующей когнитивно-дискурсивной парадигме исследований в настоящее время интерес учёных привлекает проявление авторского восприятия передаваемой информаиии и его влияние на форму изложения материала. В связи с доминирующей ролью средств массовой коммуникации становится необходимым выявление способов воздействия автора на коллективное сознание реципиентов. Особую роль в данном способе управления разумом читателей играют механизмы экспрессивного синтаксиса, выступающие ключевым звеном в передаче как объективной, так и субъективной информации. Целью исследования является выявление функиионально-лингвистических особенностей экспрессивного синтаксиса, используемого для речевого взаимодействия автора и реципиента посредством медиатекста. В рамках данной статьи проводится исследование двух англоязычных изданий, в которых с помощью метода сплошной выборки были выделены механизмы передачи категории экспрессивности на синтаксическом уровне. Для проведения системного исследования приёмов экспрессивного синтаксиса было необходимо определить особенности как самих изданий, их тематики и когнитивных установок, так и ключевые характеристики читательской аудитории, которье необходимо учитыввать при выборе экспрессивных синтаксических средств. Основным приёмом экспрессивного синтаксиса в обоих изданиях, относящихся к популярной прессе, является парентетическая конструкиия, используемая как для авторского комментария и раскрытия значения терминов или топонимов, так и для сообщения дополнительной информации. Исследование также показало, что в связи с разными когнитивными установками, типом создания (авторский или корпоративньй) и сегментами иелевой аудитории в журнале National Geographic, в отличие om The Economist, nрисутствует большее разнообразие применяемьх средств экспрессивности.

Ключевые слова: экспрессивность, экспрессивный синтаксис, медиадискурс, медиатекст, популярная пресса, парентетическая конструкция

Для цитирования: Михалчева А.В. 2021. О прагматических особенностях экспрессивного синтаксиса в текстах англоязычной популярной прессы. Филологические науки в МГИМО. Том 7. № 1(25). С. 60-67. https://doi.org/10.24833/2410-2423-2021-1-25-60-67

\section{Введение}

И зучение категории экспрессивности является одним из ключевых направлений в современной лингвистике. Такой интерес учёных обосновывается важностью данной категории в трансляции субъективной стороны речи и во влиянии на сознание адресата сообщения. И.В. Арнольд определяет экспрессивность как свойство текста, передающее смысл с «увеличенной интенсивностью» [4, с. 206]. Учёный считает, что результатом проявления экспрессивности выступает эмоциональное или логическое усиление, которое может быть образным, как в случае с использованием стилистических фигур, так и необразным, подразумевая грамматические средства интенсификации. Особую значимость в прагматическом воздействии на реципиента имеют экспрессивные синтаксические конструкции, поскольку именно единицы синтаксиса играют огромную роль в построении высказывания и передаче закладываемой объективной и субъективной информации, непосредственно соотносясь с мыслительным и коммуникативным процессами [7]. Основной целью данного исследования является описание механизмов экспрессивного синтаксиса и заложенного в них прагматического потенциала, благодаря которому становится возможным не только речевое взаимодействие автора и реципиента в рамках медиатекста, но и воздействие на сознание последнего.

Экспрессивный синтаксис можно определить как «учение о построении выразительной речи, предметом изучения которой являются лингвистические основы экспрессивной речи» [2, с. 7]. 
Основы теории экспрессивного синтаксиса заложены в работах таких учёных как Г.Н. Акимова, О.В. Александрова, Э.М. Береговская и др. Г.Н. Акимова утверждает, что синтаксический уровень языка выступает наиболее продуктивным в передаче экспрессии, ключевой характеристикой выражения которой она называет повышение выразительности за счёт присутствия стилистического эффекта и нарушения стандартного строя предложения [1]. Ведущим приёмом экспрессивного синтаксиса учёный называет конструкции расщеплённого синтаксиса, нарушающие привычный порядок слов и изменяющие просодический рисунок высказывания, делая его экспрессивным.

О.В. Александрова считает, что ключевым моментом изучения экспрессивного синтаксиса является рассмотрение синтаксических связей как в предложении, так и в их взаимодействии в рамках сверхфразового единства, благодаря чему учёный выделяет несколько уровней синтаксической экспрессии. В зависимости от возникающей между элементами синтаксической связи, учёный выделяет уровень словосочетаний, уровень компонентов актуального членения предложения и уровень базовых и парентетических конструкций [2]. Несмотря на разные синтаксические связи и разную роль в предложении, О.В. Александрова доказывает, что выделяемые уровни существуют в комплементарности, то есть дополняют друг друга в процессе их реализации в речи.

Э.М. Береговская в рамках экспрессивного синтаксиса уделяет особое внимание изучению пунктуации и конструкций, образованных по принципу симметрии/асимметрии, к которым она относит хиазм, зевгму и градацию [5]. Учёный отмечает, что необходимо проводить анализ не только формы экспрессивных синтаксических фигур, но и учитывать их семантику, которая может оказаться ключом к правильному пониманию транслируемой фактической и прагматической информации.

Материалом исследования вышеупомянутых подходов выступали тексты художественного стиля. В рамках установившейся в современной лингвистике когнитивно-дискурсивной парадигмы [3] изучение экспрессивного синтаксиса получает новые черты. Объектом исследования становится не текст, а дискурс во всех особенностях его функционирования. В последние десятилетия огромное внимание стало уделяться изучению именно дискурса СМИ или медиадискура.

Т.Г. Добросклонская проводит масштабные исследования текстов англоязычных СМИ с целью определения ключевых особенностей выделяемого ею медиадискурса, к которым она относит размытие стилевых границ, распространение норм устной речи и тиражирование ошибок. Ключевой особенностью медиатекста Т.Г. Добросклонская выделяет отражение самых незначительных изменений речеупотребления и их распространение из-за охвата огромной аудитории и проникновения в практически любую сферу деятельности человека [6].

\section{Методы исследования}

В связи с увеличивающейся ролью и воздействием средств массовой информации на жизнь людей и развитие литературного языка становится очевидным, что необходимо проследить, каким образом медиадискурс воздействует на сознание реципиента, с помощью каких средств автору удаётся задействовать когнитивную и эмоциональную сферы личности своих читателей. Именно поэтому в данной статье будет произведена попытка комплексного анализа конструкций экспрессивного синтаксиса и их роль в воздействии на реципиента. В качестве материала исследования были использованы статьи англоязычных журналов National Geographic и The Economist, объединённые общей темой «Экология». Основными применяемыми методами исследования были выбраны метод сплошной выборки, позволяющий выделить в тексте приёмы экспрессивного синтаксиса, метод контекстологического анализа, благодаря которому становится возможным понять влияние контекста на раскрытие прагматического смысла экспрессивной синтаксической конструкции, и метод сопоставительного анализа, с помощью которого будет произведено сравнение применяемых средств экспрессивного синтаксиса в двух изданиях. Перечисленные методы позволяют не только выделить приёмы экспрессивного синтаксиса, но и проследить закономерность их применения, раскрыть их прагматический потенциал, а также выявить коммуникативные интенции автора. 


\section{Когнитивные установки \\ анализируемых изданий}

Прежде чем перейти к непосредственному анализу конструкций экспрессивного синтаксиса, выбранных методом сплошной выборки, необходимо кратко охарактеризовать исследуемые издания и их читательскую аудиторию. Журнал National Geographic является официальным изданием Национального географического сообщества. Издание совмещает в себе развлекательную и просветительскую деятельность, повествуя как о достижениях науки и интересных фактах о нашей планете, так и о печальных последствиях тяжёлых военных конфликтов или противостояний между различными национальностями. Лингвистическое наполнение статей дополняют различные визуальные средства: схемы, диаграммы, графики, отражающие основные этапы развития описываемых в статье событий. Огромную роль в издании играют фотографии, которые важны как в передаче эмоций главных героев статей, так и в создании эстетического эффекта для читателей. К целевой аудитории данного журнала относятся обычные люди, которые интересуются окружающим миром и достижениями науки, имеющими важное значение для жизнедеятельности человека. Удовлетворяя познавательный интерес своих читателей, авторы статей воздействуют на их сознание, провоцируя деятельность или хотя бы размышления о том, как сделать наш мир лучше.

Издание The Economist изначально было создано для оппозиции государственной политике. В современном виде в журнале освещаются мировые экономические и политические события. Аналитика в этих журналах содержит не только проверенные факты и цитаты довольно значимых в своей сфере деятельности людей, но и отражает общую для редакции точку зрения, передаёт их мнение и оценку. В отличие от National Geographic, где иллюстрации занимают большое место в общем объёме каждого выпуска, в журнале The Economist визуальным средствам уделяется достаточно скромная роль: в статьях представлен либо карикатурный образ описываемых событий, либо проиллюстрирован предмет, имеющий особое значение в раскрытии смысла статьи. Целевой аудиторией издания являются люди, деятельность которых связана с экономической и политической сферой, бизнесмены, и все читатели, интересующиеся мировыми событиями. Большая часть выпуска посвящена информационно-аналитической справке, но выделяются две рубрики (Science and Technology и Books and Arts), которые так же, как и National Geographic, освещают актуальное развитие научных достижений или отражают состояние современных культурных мероприятий и событий.

Как видно из характеристики двух англоязычных изданий, в них отражаются разные когнитивные установки, они ориентированы на определённую целевую аудиторию. Более того, можно утверждать, что доминирующей функцией текстов The Economist является информирование читателей, в то время как статьи National Geographic направлены как на информирование, так и на воздействие на своих реципиентов, что реализуется различными средствами трансляции информации: не только лингвистическими, но и визуальными. Именно благодаря выделению таких различий, необходимо проследить, посредством каких средств происходит воздействие на читателей, как авторы используют средства языка для прагматического влияния на читателя.

\section{Функционально-лингвистический анализ приёмов экспрессивного синтаксиса}

Проанализируем две статьи, объединённые темой «Экология». В журнале National Geographic была выбрана статья «Last Ice». B статье автор пытается привлечь внимание общественности к проблеме таяния ледников вследствие глобального потепления. Автор на примере изменения внешнего вида ледников Арктики пытается донести до читателей неизбежный печальный конец, если не будут предприняты действия со стороны правительств разных государств и международных организаций по защите окружающей среды. Рассмотрим, посредством каких экспрессивных 
средств синтаксиса автор пытается воздействовать на читателя и передать своё видение сложившейся ситуации.

Стоит отметить, что доминирующим приёмом синтаксической экспрессивности в данной статье выступает использование парентетического внесения.

1. They've just jumped into a lead - a long fissure of open water in the frozen sea [8, c. 98].

2. For reasons that scientists don't yet understand, marine mammals have tended to retain the same number of chromosomes - a key requirement for hybridization - as they diverged into different species and even genera $[8$, c. 109].

3. Right now, for example, Siberian ice contaminated with nickel and lead from the Russian industrial city of Norilsk - one of the world's most polluted places - sometimes drifts into the Canadian Arctic. There it poisons the food web as it melts [8, c. 109].

В данных примерах автор использует парентезу для раскрытия определённых терминов (1), внесения дополнительных специальных знаний (2) и объяснения топонима (3). В первом примере вставная конструкция раскрывает понятие 'lead', что направлено на расширение кругозора читателей и их словарного запаса, так как данная лексическая единица является полисемантической. Во втором примере вставная конструкция содержит дополнительные данные, комментарии автора, в котором он указывает на важность информации для дальнейшего развития событий. В третьем примере парентеза не только раскрывает смысл топонима, но и объясняет выбор автором именно данного города для иллюстрации тяжёлых последствий загрязнения окружающей среды и подчёркивания их интернационального масштаба.

С помощью парентетической конструкции автор также создаёт визуальный и акустический образы описываемых явлений:

4. Then, with a frenzied whine, the helicopter's rotor blades break the spell, and we lift off, veering southwest toward our campsite on the northernmost tip of Baffin Island, Canada, about 700 miles north of Hudson Bay [8, c. 98].

5. Since then more than a million square miles has been lost - an area roughly the size of Alaska, Texas, and California combined [8, c. 98-100].

В примере (4) автор с помощью вставной конструкции описывает звук вертолёта (with a frenzied whine), что даёт читателю возможность представить себя на месте автора, почувствовать его ощущения. Предоставленная возможность сближает читателя и автора, способствует дальнейшему их общению посредством текста, усиливает прагматический потенциал статьи в целом. В (5) примере для описания масштаба экологической катастрофы, размеров растаявших ледников Антарктики, автор, ориентирующийся в основном на американских читателей, предлагает сравнить площадь повреждения поверхности с площадью нескольких американских штатов вместе взятых. Такая апелляция к воображению читателей, к визуализации карты США позволяет передать трагическое состояние ледников и призывает к действиям по прекращению пагубного воздействия человечества.

6. As the planet heats up, the summer sea ice and all the superbly adapted life it supports - the bears, the seals, the walruses, the whales, the Arctic cod, the crustaceans, the ice algae - may well vanish around Baffin [8, c. 98].

7. As we fly over the vast frozen expanse, it almost strains belief to think that we're witnessing - and with the rest of humanity, helping to cause - its demise [8, c. 98].

8. The ice that does remain in the Arctic may provide a stable environment, albeit a compact one, for organisms that depend on ice [8, c. 108].

9. An aarluk - "kills everything" - is a killer whale; a tingugliktuq - "bad liver, don't eat" - is a horned lark. But some animals, such as robins, are so new to the Arctic that Ikummaq, at least, doesn't know names for them [8, c. 108].

Данные примеры направлены на передачу последствий невмешательства человека в развитие экологической катастрофы. В (6) примере автор намеренно перечисляет все виды животных, которые могут вымереть, если лёд продолжит таять. Данное перечисление, во-первых, помогает читателю представить данных животных и растения, оценить масштаб катастрофы, а во-вторых, 
вызывает эмоциональный отклик читателя, так как некоторые из животных хорошо ему знакомы. Автор называет семь видов, чтобы передать масштаб проблемы и дать возможность читателям осознать, что это коснётся всех обитателей планеты, в том числе и их самих. В следующем примере автор во вставной конструкции акцентирует внимание читателя на общей роли человечества в разрушении окружающей среды, тем самым ещё раз побуждает читателя задуматься и постараться уменьшить своё влияние любыми доступными способами. В примере (8) автор прибегает к употреблению интенсификатора (does) и уточнения благодаря парентезе (albeit a compact one), выражая надежду на то, что, несмотря на разрушительный характер развивающейся экологической катастрофы, некоторые животные смогут адаптироваться к новым условиям жизни в новом, но достаточно маленьком по сравнению с нынешним, мире. В девятом примере автор приводит названия животных на языке эскимосов. Это делается не только для того, чтобы расширить кругозор читателей и познакомить их с другим языком. Автор поясняет, что из-за глобального изменения климатических условий уже сейчас многие животные, обитающие в других климатических зонах, переселяются в нехарактерные для них места обитания. Это свойственно и для обитателей Арктики, где неизвестные для коренного населения названия птиц (зарянки), проживающие в средней полосе, из-за изменения климата стали появляться в нехарактерных для их среды обитания местах. Это даёт читателям основание задуматься о дальнейших последствиях глобального потепления.

10. Bowheads can live 200 years or more. (One way their age has been determined is by carbon-dating old harpoon points embedded in their bodies.) [8, c. 109].

В приведённом примере вставная конструкция примечательна тем, что носит дополнительный, познавательный характер. Автор удовлетворяет любопытство читателей, рассказывая о способе измерения продолжительности жизни гренландских китов. Автор как бы отступает от основного повествования и сообщает информацию, которая не относится к теме статьи, но удовлетворяет любопытство читателей, расширяет их знания о природе.

Помимо парентетических конструкций в статье также присутствует неполный синтаксический параллелизм:

11. It's a cold and foggy June afternoon; the inlet we're walking along is still frozen. Clouds have covered the sun for days now [8, c. 108].

12. The sun is out, the weather has finally lifted, and there's barely any wind [8, c. 109].

Данные предложения выделяются из общего потока речи в статье как своим содержанием, так и структурой. В отличие от основной линии повествования, освещающей проблемы последствий глобального потепления и таяния ледников, данные предложения описывают погоду в дни пребывания автора в Арктике. Автор намеренно выделяет их с помощью параллелизма, чтобы читатели поняли, как проходило исследование, в каких условиях живут обитатели Арктики. Эти предложения, основанные на синтаксическом параллелизме, сближают читателей и автора, помогают им представить себя на месте журналиста.

Проанализировав приёмы экспрессивного синтаксиса в данной статье, можно сделать вывод об их использовании автором для акцентирования внимания своей читательской аудитории на возможных плачевных последствиях невмешательства в разрушение окружающей среды, для побуждения их к активным действиям и осознания своей роли в нынешнем экологическом состоянии планеты.

Перейдём к рассмотрению приёмов синтаксической экспрессивности, применяемых в издании The Economist. Статья «Climate Blame Game» затрагивает проблемы глобального потепления, причиной которого становятся парниковые газы. Основным приёмом синтаксической экспрессии в статье является использование парентетических конструкций.

1. At the top of Mont Blanc, Western Europe's highest mountain, instruments recorded $7 \mathrm{C}$ (the normal June temperature would be below freezing) [9, c. 58].

В этом примере парентеза используется для уточнения информации, благодаря которой читателю предоставляется возможность сравнить обычную температуру с аномальной. Такие факты помогают понять, что глобальное потепление влияет на самые высокие уголки планеты. Также 
вставная конструкция используется для раскрытия значения топонима (Mont Blanc) - чтобы сориентировать читателя в географии и подчеркнуть значимость такого температурного скачка для жизни читательской аудитории.

2. The question on many people's minds is whether these changes, and specific events like this week's temperatures in America and Europe, are caused by greenhouse gases accumulating in the atmosphere [9, $c$. 58].

Во втором примере вставная конструкция используется автором, чтобы провести аналогию с общими фактами о глобальном потеплении и примерами недавних событий, играющих непосредственную роль в жизни читателей. Благодаря обращению к реальным фактам, автор увеличивает воздействующий эффект статьи, соединяя теоретические рассуждения и реальные факты, известные читателям из средств массовой информации.

3. Since then, research of this sort, intended to study how climate change is already promoting extreme weather, has grown rapidly [9, c. 58].

Данное предложение иллюстрирует применение не только парентетической конструкции, но и использование инверсии - вынесение обстоятельства перед предикативной основой для акцентуации внимания читателей на отправной точке проведения исследований глобального изменения климата. В парентетической конструкции автор ещё раз уточняет цели исследования для облегчения понимания читателями содержания статьи.

Анализ приёмов синтаксической экспрессивности показал, что основным механизмом создания экспрессии в данной статье выступает парентетическая конструкция. Автор использует синтаксическую экспрессию в основном для уточнения какой-либо специфической информации, необходимой для раскрытия содержания статьи: раскрытия значения топонимов, соотнесения с реальными событиями, непосредственно влияющими на жизнь читателей, или повторения ранее приведённой информации.

\section{Вывод}

Согласно проведённому анализу когнитивных установок журналов, становится возможным сделать вывод о их принадлежности к серьёзной популярной прессе, предназначенной не только для информирования читателей, но и прагматического воздействия на сознание реципиентов. В связи с их нацеленностью на разные группы читателей авторы применяют различные механизмы экспрессивного синтаксиса для максимального воздействия на конкретный сегмент целевой аудитории журналов. Общим приёмом экспрессивного синтаксиса, представленным в анализируемом материале, является парентетическая конструкция, используемая для уточнения фактов или предоставления дополнительной информации. Журналы затрагивают серьёзные темы, используют сложный терминологический аппарат или описывают явления, понятные лишь специалистам конкретной сферы деятельности. Применение парентезы облегчает общее понимание специализированной лексики, раскрывая смысл терминов, топонимов, персоналий. Парентетическая конструкция также используется для передачи авторского комментария, отражения его восприятия информации или же дополнительных фактов, необходимых для понимания заложенного в статье смысла. Тем не менее, в связи с разными когнитивными установками изданий приёмы экспрессивного синтаксиса выполняют разные прагматические функции. Тексты журнала National Geographic направлены на воздействие на когнитивную сферу читателей, передают визуальные образы описываемых событий и явлений, предоставляют читателям возможность почувствовать своё присутствие и непосредственное участие в происходящем. Издание удовлетворяет интересы достаточно разнообразной читательской аудитории, отличающейся по возрастным и интеллектуальным показателям, поэтому авторам необходимо использовать такие механизмы экспрессивного синтаксиса, которые смогут оказать максимальный прагматический эффект на разный сегмент целевой аудитории. В связи с этим в статьях присутствует большее разнообразие экспрессивных средств синтаксиса, по сравнению с текстами The Economist, направленными на воздействие на более однородную аудиторию: представителей определённого круга профессий и 
имеющих общие знания и когнитивный опыт. Из этого следует, что выбор экспрессивных средств зависит не только от желаний автора как можно точнее передать своё восприятие описываемых фактов, но и от ключевых характеристик издания и его читательской аудитории.

(C) Михалчева А.В., 2021

\section{Список литературы}

1. Акимова Г.Н. Новое в синтаксисе современного русского языка / Г.Н. Акимова. М.: Высш. шк., 1994. 168 с.

2. Александрова О.В. Проблемы экспрессивного синтаксиса: На материале английского языка / О.В. Александрова. М.: Книжный дом «ЛИБРИКОМ», 2009. 216 с.

3. Александрова О. В. О контурах новой парадигмы знания в лингвистике [О.В. Александрова, Е.С. Кубрякова] // Доклады VIII Международной конференции Структура и семантика художественного текста / Под ред. Н. В. Смирнова. Спортакадемпрогресс, 1999. С. 186-197.

4. Арнольд И.В. Интерпретация художественного текста: типы выдвижения и проблема экспрессивности // Семантика. Стилистика. Интертекстуальность: Сборник статей. СПб.: Изд-во С.-Петерб. ун-та, 1999. С. 202-211.

5. Береговская Э.М. Очерки по экспрессивному синтаксису / Э.М. Береговская. М.: Рохос, 2004. 208 с.

6. Добросклонская Т.Г. Вопросы изучения медиатекстов (опыт исследования современной английской медиаречи) / Т.Г. Добросклонская. 2014. 288 с.

7. Золотова Г.А. Очерк функционального синтаксиса русского языка / Г.А. Золотова. М.: Наука, 1973. 352 с.

8. Climate Blame Game // The Economist. Vol. 432. №9153. PP. 58-59.

9. Folger T. Last Ice // National Geographic. Vol. 233. №1. PP. 90-109.

\section{References}

1. Akimova, G.N. Novoe v sintaksise sovremennogo russkogo iazyka [New in the syntax of the modern Russian language] / G.N. Akimova. M.: Vyssh. shk., 1994. 168 p.

2. Aleksandrova, O.V. Problemy ekspressivnogo sintaksisa: Na materiale angliyskogo iazyka [On the problem of expressive syntax: based on the English language] / O.V. Aleksandrova. M.: Knizhny dom «LIBRIKOM», 2009. 216 p.

3. Aleksandrova, O. V., Kubryakova, E. S. O konturakh novoi paradigmy znaniia v lingvistike [On the contour of new paradigm of knowledge in linguistics] // Doklady VIII Mezhdunarodnoj konferencii Struktura i semantika hudozhestvennogo teksta [Presentations at the V111 International Conference Structure and Semantics of a Literary text] / Pod red. N. V. Smirnova. Sportakademprogress, 1999. P. 186-197.

4. Arnold, I.V. Interpretatsia hudozhestvennogo teksta: tipy vydvizheniia i problema ekspressivnosti [The interpretation of fiction: types of foregrounding and the problem of expressivity] // Semantika. Stilistika. Intertekstual'nost': Sbornik statej. [Semantics. Stylistics. Intertextuality. Collection of articles] SPb.: Izd-vo S.-Peterb. un-ta, 1999. P. 202-211.

5. Beregovskaya, E.M. Ocherki po ekspressivnomu sintaksisu [Essays on expressive syntax] / E.M. Beregovskaya. M.: Rohos, 2004. $208 \mathrm{p}$.

6. Dobrosklonskaya, T.G. Voprosy izucheniia mediatekstov (opyt issledovaniia sovremennoy angliiskoi mediarechi) [Studying media texts (experience of contemporary English media language study: monograph)] / T.G. Dobrosklonskaya. 2014. 288 p.

7. Zolotova, G.A. Ocherk funkcional'nogo sintaksisa russkogo iazyka [Essay on the functional syntax of the Russian language] / G.A. Zolotova. M.: Nauka, 1973. 352 p.

8. Climate Blame Game // The Economist. Vol. 432. №9153. PP. 58-59.

9. Folger, T. Last Ice // National Geographic. Vol. 233. №1. PP. 90-109.

\section{Сведения об авторе:}

Михалчева Анастасия Витальевна - ассистент кафедры английской филологии ТГПУ им. Л.Н. Толстого (Тула, Россия). Сфера научных и профессиональных интересов: грамматика английского языка, категория экспрессивного синтаксиса, медиадискурс. E-mail: anastasia-mikhalcheva@rambler.ru

\section{About the author:}

Anastasia V. Mikhalcheva - Assistant of the Department of English Philology in Tula State Lev Tolstoy Pedagogical university (Tula, Russia). Spheres of research and professional interest: English language grammar, the category of expressive syntax, media discourse. E-mail: anastasia-mikhalcheva@rambler.ru 